\title{
Understanding the extracellular matrix in acute myeloid leukemia
}

\author{
Valerio Izzi, ${ }^{1}$ Ritva Heljasvaara ${ }^{1,2}$ and Taina Pihlajaniemi ${ }^{1}$ \\ ${ }^{1}$ Centre of Excellence in Cell-Extracellular Matrix Research and Biocenter Oulu, Faculty of Biochemistry and Molecular Medicine, \\ University of Oulu, Finland and ${ }^{2}$ Centre for Cancer Biomarkers (CCBIO), Department of Biomedicine, University of Bergen, Norway
}

E-mail:valerio.izzi@oulu.fi

doi:10.3324/haematol.2017.174847

$\mathrm{D}$ espite the continuous progress in therapy, acute myeloid leukemia (AML) remains the leading cause of death among hematologic neoplasms. Understanding how leukemic clones arise and how they interact with the microenvironment of the bone marrow, especially within the hematopoietic stem cell (HSC) niches, is thus necessary to provide the means to early diagnosis and intervention. In this context, much has been published about the influence of niche stromal cell-derived extracellular matrix (ECM) on leukemic stem cells (LSCs, the apex of the neoplastic progeny which is AML itself), ${ }^{2,3}$ but considerably less is known about whether LSCs directly produce their 'own' ECM to hijack niche functions and gain a growth advantage over HSCs. Hence, we investigated the expression of ECM genes in both LSCs (and, more generally, leukemic precursor cells) and mature AML cells as compared to normal HSCs and white blood cells from healthy donors. We found that a common set of 80 ECM genes (either up- or down-regulated with respect to healthy donors) characterizes all leukemic cells, independently from specific cytogenetic alterations or mutations in driver genes, and constitutes the ECM signature of the whole leukemic process from the LSCs to the final circulating blasts ${ }^{4}$ (Figure 1).

Our results, together with those of a few others, suggest a much more active role for LSCs in the production of a modified ECM in the niche, one that favors disease progression. These data will help us to decipher the relationships between AML and the microenvironment, and will hopefully result in new diagnostic and therapeutic possibilities in the future.

In 1889, Stephen Paget proposed the visionary "seed and soil" theory, a conjecture which acknowledged the importance of a permissive microenvironment (the bad soil) in the growth of neoplastic cells (the bad seeds). ${ }^{5}$ Approximately 100 years later, the works by Schofield and Kimble \& White changed the prevailing vision of stem cells (SCs), showing for the first time that elements other than SCs control and influence the growth and fate of these cells. ${ }^{6}$ Today we define the stem cell niches as specialized anatomical regions in which a complex network of stromal cells and ECM interact with each other and with the SCs as a 'dynamic duo', and we know that SCs and cancer are two sides of the same coin, with the normal SCs and their niches on one side and the cancer SCs (CSCs) and their niches on the other., ${ }^{2,3}$

The ECM provides several types of microenvironmental cues that sustain SCs. Due to its ability to seize and present cytokines and growth factors, provide an adhesive substrate for the cells, and to generate and integrate the mechanical signals needed to control cell proliferation and differentiation, the ECM is, in fact, a critical determinant of the properties and behavior of the cells embedded into it. ${ }^{8}$ HSCs and their niches are no exceptions to this rule, and a substantial amount of data now point to the importance of sensing and correctly interacting with the proper ECM in the bone marrow for the HSCs to lodge in their niches and accomplish their functions. ${ }^{9}$

It is all the more surprising, then, to notice that, even though the very definition of CSCs came from an AML study, ${ }^{10}$ research on LSCs has taken a rather unidirectional road. While findings on genetic and epigenetic networks controlling LSCs have accumulated rapidly, and the importance of stromal elements and altered ECM in the growth and dissemination of leukemic cells is nowadays well accounted for, ${ }^{2,3,5}$ there seems to be a paucity of results to explain how far the leukemic cells themselves contribute to the changes occurring in the niche. For example, it is widely accepted that the expression of the major cellular ECM receptors, the integrins, on LSC allows fibronectin sensing, lodging these cells to the bone marrow niche and triggering pro-survival signal cascades that ultimately lead to post-therapy persistence of the leukemic clones. ${ }^{11}$ Likewise, the expression of another cellular receptor, CD44, on LSC and AML cells allows the interaction of these cells with hyaluronan, osteopontin, fibronectin and selectins, conferring a superior engrafting ability to the malignant clones and a crucial survival mechanism. ${ }^{3,11}$

But what about the direct interference of LSCs with the niche ECM? Considering how important a 'permissive' ECM is for LSCs, we speculated that transcriptional programs enabling leukemic cells to directly alter the composition of the surrounding ECM would give them a growth advantage as they could quickly shape and hijack the microenvironment even before establishing a detrimental co-operation with stromal cells. Following this idea, we investigated the expression of ECM genes in LSC and HSC and, in parallel, in AML cells versus normal white cells. We found a core set of 80 ECM genes that were differentially regulated in leukemic cells with respect to their normal counterparts. ${ }^{4}$ Notably, our results not only largely recapitulated previous non-systematic findings, but also gave them a wider context. Thus, in cells with high CD44 expression, we observed a significant upregulation of ECM proteins which would directly interact with CD44 itself, including structural substrates, such as collagen IV and XVIII and laminin beta 2 (COL4A5, COL18A1 and LAMB2, respectively), and proteinases implicated in ECM assembly, remodeling and growth factor activation, such as matrix metalloproteinase 2, ADAM metallopeptidase domain 17, bone morphogenetic protein 1 and cathepsin G (MMP2, ADAM17, BMP1 and CTSG, respectively). ${ }^{4}$

Building on these data, we found that ECM gene expression in leukemic cells is not univocal and that the differential 


\section{The ECM signature of LSCs and AML}
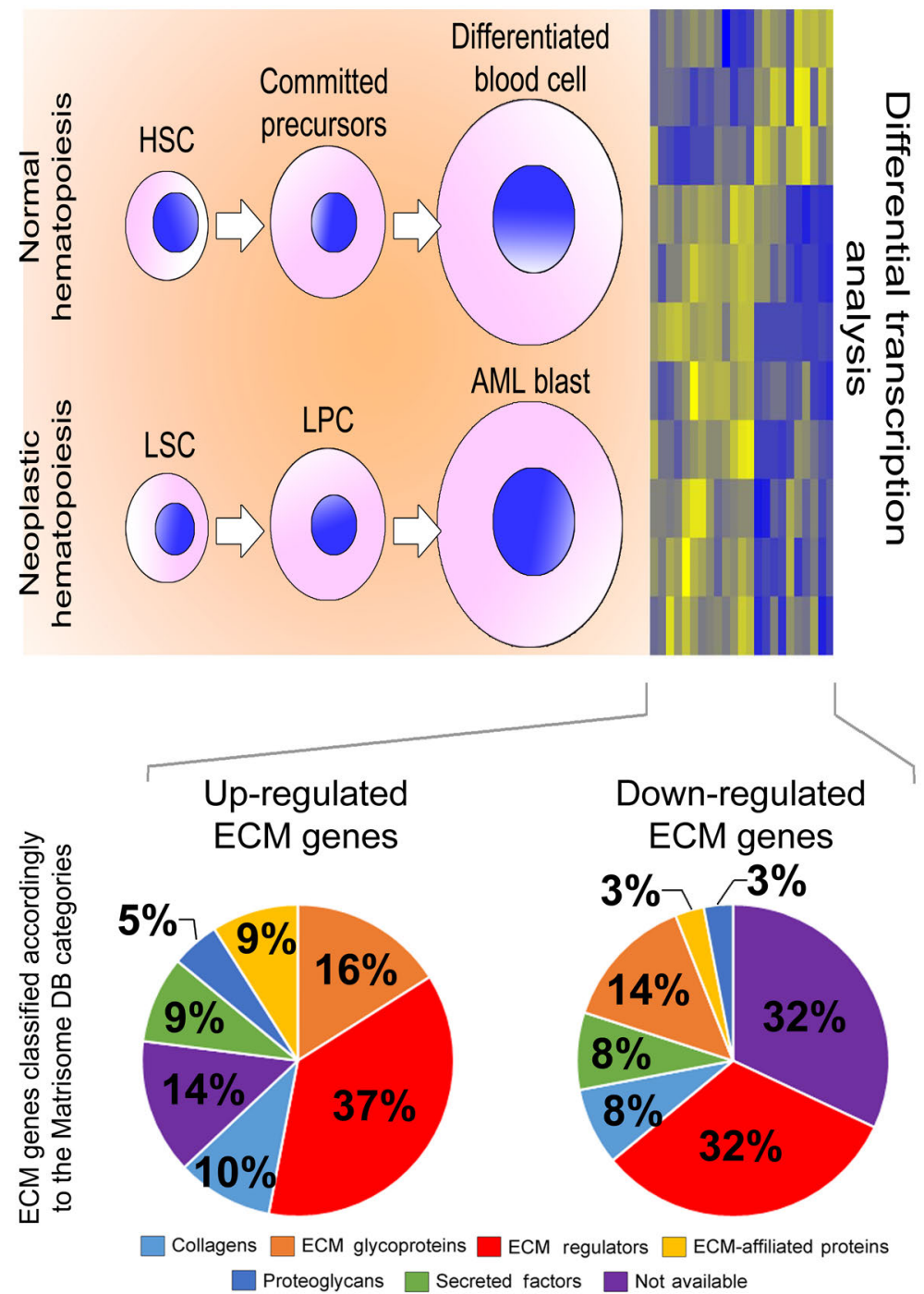

Figure 1. The extracellular matrix (ECM) signature of leukemic stem cells (LSCs) and acute myeloid leukemia (AML). Compared to normal hematopoietic stem cells (HSC), committed precursors or differentiated blood cells, the expression of ECM genes in LSC, leukemia precursor cells (LPC) or AML blasts differs significantly, and spans all the major categories of ECM components, enzymes and secreted factors represented in the Matrisome DB database. Note that some ECM genes are not present in the Matrisome DB and are reported as "Not available".

expression of a restricted set of these genes distinguishes two subtypes of leukemic precursor cells not previously reported: one subtype, clustering with normal HSCs with respect to the overall ECM expression profile (we named them "early leukemic" group), and another with a clearly distinguishable profile (we termed these cells "definitive leukemic"). When these two profiles were used to classify AML patients, we found that individuals with the "definitive leukemic" profile had much lower overall, diseasefree and event-free survival independently of karyotype aberrations or typical driver mutations, for example, in FLT3, NPM1 or IDH1 genes. ${ }^{4}$

Recently, Foroushani et al. provided another important confirmation of the view that AML actively creates its own ECM. They employed a sophisticated network analysis to identify the architecture of active gene regulatory networks in AML and found that the regulation of ECM modules is the most significant feature of AML transcriptional profile. ${ }^{12}$ Of particular interest is the downregulation of matrix metalloproteinase 9 (MMP9) observed in both Foroushani et al.'s and our studies; a finding in contrast with the general idea that $M M P 9$ is highly expressed in AML. ${ }^{13}$ Yet, our systematic approach suggests a possible explanation to this. In fact, we found that $M M P 9$ belongs to a specific subnetwork of ECM elements interacting with CD44, and that the expression of CD44 characterizes patients with lower survival. ${ }^{4}$ If we then consider that a previous report suggested that $M M P 9$ levels correlate inversely with patients' risk of death, ${ }^{14}$ we can easily envisage a regulatory mechanism that, in this CD44 subnetwork, transcribes genes that facilitate the spreading of AML while suppressing genes (such as MMP9) that would halt it.

Altogether, these findings lay the foundation for a more systematic analysis of the direct ECM-modifying activities of LSC and AML cells, and support a much more active role for these cells in the regulation of the niche ECM than had been previously supposed. 


\section{References}

1. Adult Acute Myeloid Leukemia Treatment (PDQ®)-Health Professional Version 2017 [updated 20.1.2017; cited 15.6.2017]. Available from: https://www.cancer.gov/types/leukemia/hp/adultaml-treatment-pdq.

2. Plaks V, Kong N, Werb Z. The cancer stem cell niche: how essential is the niche in regulating stemness of tumor cells? Cell Stem Cell. 2015;16(3):225-238

3. Schepers K, Campbell TB, Passegue E. Normal and leukemic stem cell niches: insights and therapeutic opportunities. Cell Stem Cell. 2015;16(3):254-267

4. Izzi V, Lakkala J, Devarajan R, et al. An extracellular matrix signature in leukemia precursor cells and acute myeloid leukemia. Haematologica. 2017;102(7):e245-e248.

5. Langley RR, Fidler IJ. The seed and soil hypothesis revisited--the role of tumor-stroma interactions in metastasis to different organs. Int J Cancer. 2011;128(11):2527-2535

6. Albert Hubbard EJ. The C. elegans germ line: a model for stem cell biology. Dev Dyn. 2007;236(12):3343-3357

7. Voog J, Jones DL. Stem cells and the niche: a dynamic duo. Cell Stem Cell. 2010;6(2):103-115
8. Ahmed M, Ffrench-Constant C. Extracellular Matrix Regulation of Stem Cell Behavior. Curr Stem Cell Rep. 2016;2:197-206.

9. Krause DS. Regulation of hematopoietic stem cell fate. Oncogene. 2002;21(21):3262-3269.

10. Lapidot T, Sirard C, Vormoor J, et al. A cell initiating human acute myeloid leukaemia after transplantation into SCID mice. Nature. 1994;367(6464):645-648.

11. Konopleva MY, Jordan CT. Leukemia stem cells and microenvironment: biology and therapeutic targeting. J Clin Oncol 2011;29(5):591-599.

12. Foroushani A, Agrahari R, Docking R, et al. Large-scale gene network analysis reveals the significance of extracellular matrix pathway and homeobox genes in acute myeloid leukemia: an introduction to the Pigengene package and its applications. BMC Med Genomics. 2017;10(1):16

13. Travaglino E, Benatti C, Malcovati L, et al. Biological and clinical relevance of matrix metalloproteinases 2 and 9 in acute myeloid leukaemias and myelodysplastic syndromes. Eur J Haematol. 2008;80(3):216-226.

14. Schmohl J, Santovito D, Guenther T, et al. Expression of surface-associated 82kDa-proMMP-9 in primary acute leukemia blast cells inversely correlates with patients' risk. Exp Hematol. 2016;44(5):358-362.e5. 